\title{
Adenovirus-mediated ING4 expression reduces multidrug resistance of human gastric carcinoma cells in vitro and in vivo
}

\author{
ZONG-LEI MAO ${ }^{1^{*}}$, SONG-BING HE ${ }^{1,2^{*}}$, WEI-HUA SHENG ${ }^{3}$, XIAO-QIANG DONG ${ }^{1}$ and JI-CHENG YANG ${ }^{3}$ \\ ${ }^{1}$ Department of General Surgery, The First Affiliated Hospital of Soochow University, Suzhou, Jiangsu 215006; \\ ${ }^{2}$ Shanghai Institute of Immunology, Shanghai Jiao Tong University School of Medicine, Shanghai 200025; \\ ${ }^{3}$ Cell and Molecular Biology Institute, College of Medicine, Soochow University, Suzhou, Jiangsu 215006, P.R. China
}

Received July 5, 2013; Accepted August 11, 2013

DOI: $10.3892 /$ or.2013.2671

\begin{abstract}
Chemotherapy is the primary treatment for both resectable and advanced gastric carcinoma, yet multiple drug resistance (MDR) of gastric carcinoma remains a significant therapeutic obstacle. The development of novel strategies to reduce MDR in gastric carcinoma would yield a better outcome following chemotherapy. ING4, a member of the inhibitor of growth (ING) tumor-suppressor family, possesses antitumor and radiosensitization or chemosensitization effects in a variety of human cancers. The present study investigated the effects and possible mechanisms of action of adenovirus-mediated ING4 (AdVING4) on the reversion of human gastric carcinoma cell MDR in vitro and in vivo in nude mouse xenografts. The data showed that the expression of ING4 mRNA and protein was dramatically downregulated (or lost) in gastric carcinoma SGC7901/CDDP cells after CDDP-induced MDR phenotype and in the parental SGC7901 cells. AdVING4-induced ING4 expression reversed MDR and induced apoptosis of SGC7901/CDDP cells in vitro and in vivo in the SGC7901/CDDP xenograft tumors. Furthermore, AdVING4 substantially downregulated the expression of MDR-related proteins P-gp and MRP1 and apoptosis-related proteins Bcl-2 and survivin, but upregulated the expression of apoptosis-related protein Bax in the SGC7901/CDDP xenograft tissues. The reversion effects elicited by AdVING4 on gastric cancer cell MDR were closely associated with the downregulation of ATP-binding cassette transporters and
\end{abstract}

Correspondence to: Dr Ji-Cheng Yang, Cell and Molecular Biology Institute, College of Medicine, Soochow University, 188 Shizi Street, Suzhou, Jiangsu 215006, P.R. China

E-mail: jcyang@suda.edu.cn

Dr Xiao-Qiang Dong, Department of General Surgery, The First Affiliated Hospital of Soochow University, Suzhou, Jiangsu 215006, P.R. China

E-mail: captain_hsb@163.com

*Contributed equally

Key words: chemotherapy, multiple drug resistance, inhibitor of growth, gastric carcinoma activation of apoptotic pathways. Thus, these findings suggest that AdVING4 may be a feasible modulator for the MDR phenotype of gastric carcinoma cells.

\section{Introduction}

Gastric cancer ranks as the third and fifth most common malignancy in males and females, respectively, in the world (1). Although its morality has significantly declined in recent years, gastric cancer is still estimated to account for approximately $10 \%$ of invasive cancers with a high incidence in Japan, China, Korea, Central and South American (2-5). To date, chemotherapy remains the primary treatment option for both resectable and advanced gastric cancer to improve overall survival and the quality of life of patients $(6,7)$. However, expression of multiple drug resistance (MDR) genes is a major obstacle to successful chemotherapy of various blood-related and solid tumors (8), including gastric cancer. It has been recognized that during or following chemotherapy, MDR occurs, and the underlying molecular mechanisms have been extensively studied in vivo and in vitro (9-12). For example, upregulation of ATP-binding cassette transporters, such as P-glycoprotein (P-gp) and MDR-associated protein 1 (MRP1) are mainly responsible for chemotherapy-induced MDR. Recently, several novel factors acting downstream of the initial drug-induced insult have been shown to play an important role in the development of MDR, such as enhanced DNA repair activity, defective apoptosis pathway, or altered metabolism of drugs (10). The MDR mechanisms in gastric carcinoma cells have been extensively explored, but there are no clear answers. Accumulated evidence to date suggests that mechanisms responsible for MDR in gastric carcinoma are likely to be extremely intricate. Therefore, there is a need to further explore the mechanisms involved in MDR and to identify efficient and low toxicity modulators to be used for the clinical treatment of gastric cancer.

Inhibitor of growth 4 (ING4) represents a novel member of the ING tumor-suppressor family, which was isolated and characterized by Shiseki et al in 2003 (13). The tumor-suppressor protein ING4 can interact with p53, inhibit cell growth and induce apoptosis in different types of cells. ING4 protein contains a plant homeodomain (PHD)-finger motif (14), a transcriptional regulator that involves chromatin remodeling (15), 
in its $\mathrm{COOH}$-terminal region and a potential bipartite nuclear localization signal (NLS) (16) in its middle region. More recently, ING4 has been shown to play a role in the regulation of DNA repair, tumor growth, angiogenesis, migration and gene transcription. Furthermore, ING4 can suppress the loss of contact inhibition induced by myelocytomatosis viral-related oncogene, neuroblastoma derived (MYCN) and myelocytomatosis viral oncogene homolog (MYC) family oncogenes (17). In addition, the ING4-dominant mutant can promote MYC-initiated mouse mammary carcinogenesis (18), whereas overexpression of ING4 protein can apparently enhance tumor growth inhibition in a p53-dependent and p53-independent manner by induction of cell cycle alteration and apoptosis $(13,19-21)$ and inhibition of tumor angiogenesis by repressing nuclear factor $\kappa \mathrm{B}$ and hypoxia-inducible factor-1 $\alpha$ (22-24). In addition, ING4 can enhance radiosensitivity and chemosensitivity in non-small cell lung cancer SPC-A1 cells and hepatocarcinoma cells, respectively $(19,25)$. Previous studies have shown that the combination of radiotherapy, chemotherapy, and other conventional therapies with gene therapy is a promising practice for the treatment of cancers (26-28). ING4 has been reported to enhance chemosensitivity to DNA-damage agents, such as doxorubicin or etoposide in human hepatocarcinoma cells $(19,26)$. Our previous data also demonstrated that adenovirus-mediated (AdVING4) gene therapy sensitized human hepatocarcinoma cells to cisplatin (CDDP) and enhanced the radiosensitivity of non-small-cell lung cancer $(19,25)$. However, reversal of its resistance in human cancers has not been reported. Therefore, on the basis of prior studies, we hypothesized that ING4 may affect the MDR phenotype of human gastric carcinoma cells. In the present study, we investigated the possible role of ING4 in the reversion of human gastric cancer cell MDR and its underlying mechanisms.

\section{Materials and methods}

Adenoviruses, cell lines, reagents and mice. The AdVING4 and AdV-green fluorescent protein (AdVGFP) replicationincompetent Ad5E1- and E3-deleted adenoviruses were previously constructed in our laboratory (21). A human embryonic kidney cell line QBI-293A was a kind gift from Professor Jiang Zhong of Fudan University (Shanghai, China). The human gastric carcinoma cell line SGC7901 was purchased from the American Type Culture Collection (ATCC; Rockville, MD, USA). QBI-293A and SGC7901 cell lines were cultured in RPMI-1640 medium (Gibco-BRL, Shanghai, China) supplemented with $10 \%$ fetal bovine serum (FBS) (HyClone, Logan, UT, USA). TRIzol reagent and the reverse transcriptase MuMLV were purchased from Invitrogen (Shanghai, China). The Cell Counting Kit- 8 was purchased from the subsidiary of Dojindo Laboratories (Shanghai, China). A polyclonal anti-ING4 antibody was purchased from Abcam (Shanghai, China). The SuperEnhanced chemiluminescence detection kit was obtained from Applygen Technologies Inc. (Beijing, China). The antibodies against P-gp, MRP1, Bax, Bcl-2, and survivin were from Cell Signaling Technology, Inc. (Boston, MA, USA). An UltraSensitive ${ }^{\mathrm{TM}}$ SP kit was obtained from Maixin (Fuzhou, China). Chemotherapeutical drugs, cisplatin (CDDP), 5-fluorouracil (5-FU) and adriamycin (ADM), were kindly provided by The First Hospital Affiliated to Soochow University (Suzhou, China). In addition, female athymic nude mice were purchased from the Shanghai Experimental Animal Center (Shanghai, China) and maintained in the animal facility at Soochow University according to the guidelines of the Animal Research Committee of Soochow University.

Establishment of the CDDP-based MDR phenotype in the human gastric carcinoma cell line SGC7901/CDDP. Gastric cancer SGC7901 cells were cultured in RPMI-1640 supplemented with $10 \%$ FBS overnight, and the growth medium was replaced with medium containing $0.05 \mu \mathrm{g} / \mathrm{ml}$ cisplatin to induce cells to MDR following a gradual increase in the drug concentrations to $2 \mu \mathrm{g} / \mathrm{ml}$. After 6 months of culture, the SGC7901 cells were able to stably grow in the growth medium containing $2 \mu \mathrm{g} / \mathrm{ml}$ cisplatin. To maintain the MDR phenotype, the medium containing $2 \mu \mathrm{g} / \mathrm{ml}$ cisplatin was used in the subsequent experiments.

Infection of the AdVING4 virus to induce ING4 expression in gastric cancer cells. AdVING4 and control AdV adenoviruses were prepared as described previously (21). The titer of the purified adenoviruses was determined by using the gene transfer unit method (GTU) through calculation of the numbers of the reporter gene GFP-expressing QBI-293A cells in $18 \mathrm{~h}$ of adenoviral infection using fluorescence microscopy. To assess the optimal ratio of infectious adenovirus (GTU) to target cells, a multiplicity of infection (MOI), for a maximal infection and transgene expression, human gastric carcinoma cells SGC7901 and SGC7901/CDDP were infected with AdVING4 and AdV at various MOIs (0, 10, 25, 50, 100, 150 and 200, respectively) for $24 \mathrm{~h}$. The adenoviral infection efficiency was detected according to the GFP expression. Furthermore, the ING4 transgene expression meditated by the adenoviral infection in SGC7901 and SGC7901/CDDP gastric carcinoma cells was determined using reverse transcriptase-PCR and western blot analysis.

RNA isolation, reverse transcriptase-PCR and $q R T-P C R$. Total cellular RNA was isolated from AdVING4- or AdV-infected and uninfected SGC7901 and SGC7901/CDDP cells using TRIzol and then reversely transcribed into cDNA using oligo $\mathrm{d}(\mathrm{T})_{18}$ as primer according to the manufacturers' protocols. PCR amplification was carried out using these cDNA samples as templates and ING4 primers (5'-GCGTCGA CATGGATGATGGGATGTATTTGGAAC-3' and 5'-GCAA GCTTCTATTTCTTCTTCCGTTCTTGGGAG-3') under conditions of an initial 1 cycle at $94^{\circ} \mathrm{C}$ for $2 \mathrm{~min}$ and $72^{\circ} \mathrm{C}$ for $10 \mathrm{~min}$ followed by 35 cycles at $94^{\circ} \mathrm{C}$ for $50 \mathrm{sec}, 58^{\circ} \mathrm{C}$ for $50 \mathrm{sec}$ and $72^{\circ} \mathrm{C}$ for $55 \mathrm{sec}$ and a final extension at $72^{\circ} \mathrm{C}$ for $10 \mathrm{~min}$. The PCR products were separated on $1 \%$ agarose gel using electrophoresis with ethidium bromide staining.

To determine the expression levels of MDR1, MRP1, Bcl-2, Bax and survivin mRNA in the AdVING4-transfected SGC7901/CDDP cells, qRT-PCR based on SYBR-Green I detection was performed using MJ Research Opticon ${ }^{\mathrm{TM}}$ 2 System (MJ Research). Briefly, the total cellular RNA was isolated using TRIzol reagent and reversely transcribed into cDNA (see above). PCR primers were: MDR1, 5'-ATGCC TTCATCGAGTCACTG-3' and 5'-TAACAAGGGCACGAG 
CTATG-3'; MRP1, 5'-ATGCCTTCATCGAGTCACTG-3' and 5'-TAACAAGGGCACGAGCTATG-3'; Bcl-2, 5'-GCCCTGT GGATGACTGAGTA-3' and 5'-CAGCCAGGAGAAATCAA ACA-3'; Bax, 5'-ACGGCCTCCTCTCCTACTTT-3' and 5'-CAGCCCATCTTCTTCCAGAT-3'; survivin, 5'-TTCTCA AGGACCACCGCATC-3' and 5'-GCCAAGTCTGGCTCGT TCTC-3' and GAPDH, 5'-GCACTCGAGCCATGAATTT TCA-3' and 5'-GCTTCTAGATCAGAGCTTGT-3'. The qPCR amplification contained a total volume of $50 \mathrm{ml}$ of $1 \mathrm{ml} \mathrm{cDNA}$, $1 \mathrm{ml}$ of $900 \mathrm{nM}$ of each primer, $25 \mathrm{ml} 2$ X SYBR-Green I PCR Master mix, and $22 \mathrm{mldd}_{2} \mathrm{O}$ and was subsequently performed according to the following program: $94^{\circ} \mathrm{C}$ for $2 \mathrm{~min}$ and $72^{\circ} \mathrm{C}$ for $10 \mathrm{~min}$ followed by 35 cycles of $94^{\circ} \mathrm{C}$ for $30 \mathrm{sec}, 58^{\circ} \mathrm{C}$ for $30 \mathrm{sec}$, and $72^{\circ} \mathrm{C}$ for $30 \mathrm{sec}$. The level of expression of each gene was normalized to the internal control gene GAPDH and calculated using the pooled cDNA from all samples by the $2^{-\Delta \Delta C T}$ method as described previously (29). The authenticity of the PCR products was verified by melting curve analysis and agarose gel electrophoresis. Each sample was analyzed in duplicate in an independent reaction, and the experiment was repeated thrice.

Protein extraction and western blotting. Total cellular protein was extracted from the AdVING4- or AdV-infected and uninfected SGC7901 and SGC7901/CDDP cells and resolved in $12 \%$ sodium dodecyl sulfate-polyacrylamide gel electrophoresis (SDS-PAGE) and subsequently transferred onto a polyvinylidene difluoride membrane. Subsequently, the membrane was incubated in $5 \%(\mathrm{w} / \mathrm{v})$ non-fat dry milk in Tris-buffered saline containing 0.05\% Tween-20 (TBST) for $1 \mathrm{~h}$ at $37^{\circ} \mathrm{C}$ and then further with a primary antibody of polyclonal goat anti-ING4 $(1: 1,000)$ in blocking solution for $1 \mathrm{~h}$ at $37^{\circ} \mathrm{C}$. The membrane was washed with TBST and incubated with a peroxidase horseradish peroxidase-conjugated secondary antibody of rabbit anti-goat $\operatorname{IgG}(1: 3,000)$ in blocking solution for another $1 \mathrm{~h}$ at $37^{\circ} \mathrm{C}$. After being washed with TBST thrice, the positive bands were developed using a SuperEnhanced chemiluminescence detection kit and visualized after exposure of the membranes to Kodak X-ray film.

Cell viability $C C K-8$ assay. The in vitro resistance index of human gastric carcinoma SGC7901/CDDP cells to CDDP was determined using the CCK-8 assay. Briefly, SGC7901 and SGC7901/CDDP cells were dispensed into 96-well culture plates at $1 \times 10^{4} /$ well and incubated for $24 \mathrm{~h}$ at $37^{\circ} \mathrm{C}$ and treated with CDDP, 5-FU, ADM at 7 different concentrations (see Results) for $48 \mathrm{~h}$. The viability of SGC7901 and SGC7901/CDDP cells was analyzed using the CCK-8 kit according to the manufacturer's protocol. Similarly, the cells infected with AdVING4 or control AdV were also included in the CCK-8 assay. The SGC7901/CDDP cells were infected with 100 MOI AdVING4 or AdV or without adenovirus (PBS control) for $24 \mathrm{~h}$ and then treated with CDDP, 5-FU or ADM for $48 \mathrm{~h}$ and subjected to CCK- 8 assay. The inhibitory rate (\%) was calculated using the formula: 1 - $\left(\mathrm{OD}_{\text {experiment }} / \mathrm{OD}_{\text {control }}\right) \times 100 \%$; Resistance index: $\left.\mathrm{IC}_{50(\mathrm{SGC} 7901 / \mathrm{CDDP})} / \mathrm{IC}_{50(\mathrm{SGC} 7901)}\right)$ and Reversion index: $\mathrm{IC}_{50(\mathrm{AdVING} 4)} / \mathrm{IC}_{50(\mathrm{PBS})}$.

Nude mouse xenograft assay. To test the effects of ING4 expression in vivo, we performed an animal experiment, which divided animals into 7 groups [PBS + SGC7901 $\left(\mathrm{PBS}_{1}\right)$, PBS + SGC7901/CDDP ( PBS $\left._{2}\right)$, AdV + SGC7901/CDDP (AdV), AdVING4 + SGC7901/CDDP (AdVING4), CDDP + SGC7901/CDDP (CDDP), AdV + CDDP + SGC7901/CDDP (AdV + CDDP) and AdVING4 + CDDP + SGC7901/CDDP (AdVING4 + CDDP)]. Briefly, female athymic nude mice were subcutaneously (s.c.) inoculated in the armpits of their right anterior limbs with $2 \times 10^{6}$ human gastric carcinoma SGC7901 cells $\left(\mathrm{PBS}_{1}\right.$ ) and SGC7901/CDDP (the other group). After the tumor mass reached a mean tumor volume of $\sim 100-120 \mathrm{~mm}^{3}$, AdVING4, AdV or PBS was administered once every 3 days by intratumoral injection for 36 days. From days 7 to 14 and 21 to $28,4.5 \mathrm{mg} / \mathrm{kg}$ of CDDP was administered weekly via tail vein injection. Tumor progression and regression were monitored, and tumor volume was measured with a caliper every 4 days. The tumor volume was calculated by the formula, $\mathrm{ab}^{2} / 2$, where $a$ is the larger and $b$ is the smaller of the 2 dimensions of the tumor mass. The tumor-bearing mice were then sacrificed at day 36 after the treatments and tumor xenograft tissues were removed, weighed, fixed by $10 \%$ neutral formalin, and then embedded in paraffin for hematoxylin and eosin staining and immunohistochemical analysis.

Immunohistochemistry. Expression of P-gp, MRP1, Bcl-2, $\mathrm{Bax}$, and survivin proteins in human gastric carcinoma xenograft tissues was analyzed using immunohistochemistry with an UltraSensitive ${ }^{\mathrm{TM}}$ SP kit according to the manufacturer's instructions. The presence of buffy or brown diaminobenzidine precipitates is indicative of positive reactivity. The integral optical density (IOD) of immunohistochemical intensity was analyzed by Image-Pro Plus 6.0 software (Media Cybernetics, Bethesda, MD, USA).

Statistical analysis. All data are presented as the means \pm SD. The significant difference between 2 groups was evaluated using the Student's t-test and one-way or two-way repeated measures analysis of variance and multiple comparisons with SPSS 10.0 software (SPSS, Chicago, IL, USA). A value of $\mathrm{P}<0.05$ was considered to indicate a statistically significant result.

\section{Results}

Establishment of the gastric carcinoma SGC7901/CDDP cell line for CDDP-induced MDR phenotype. To obtain the CDDP-induced MDR phenotype in gastric cancer SGC7901/CDDP cells, the cells were grown under increasing cisplatin concentrations from 0.05 to $2 \mu \mathrm{g} / \mathrm{ml}$ during a 6 month period of time. The CDDP-resistant SGC7901 cells were able to grow stably in $2 \mu \mathrm{g} / \mathrm{ml}$ cisplatin-containing medium. To maintain the MDR phenotype, $2 \mu \mathrm{g} / \mathrm{ml}$ of cisplatin-containing medium was used for all the experiments. Cell viability CCK-8 assay was performed initially to detect the altered cell viability in the SGC7901/CDDP cells. The data showed that the SGC7901/CDDP cells acquired 6.68-, 7.53- and 19.92fold resistance to ADM, 5-FU and CDDP compared to the parental SGC7901 cell line, respectively (Fig. 1A). Moreover, expression of MDR-related genes (MDR1, MRP1, and apoptosis-related Bcl-2, survivin genes) was apparently upregulated while the apoptosis-related Bax gene was downregulated in 
A

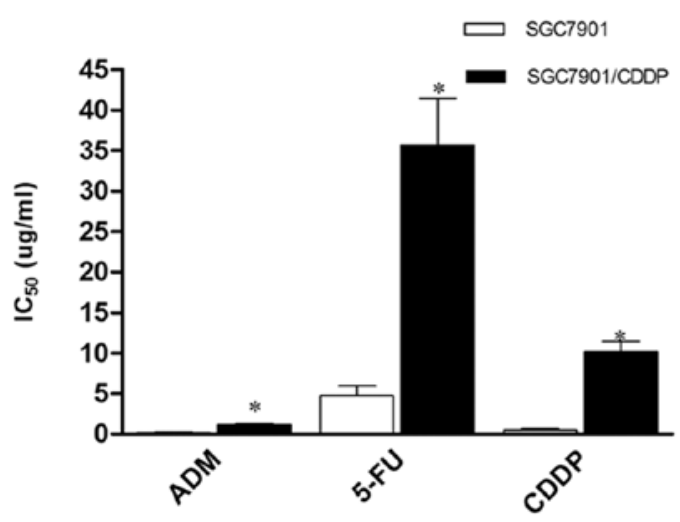

B

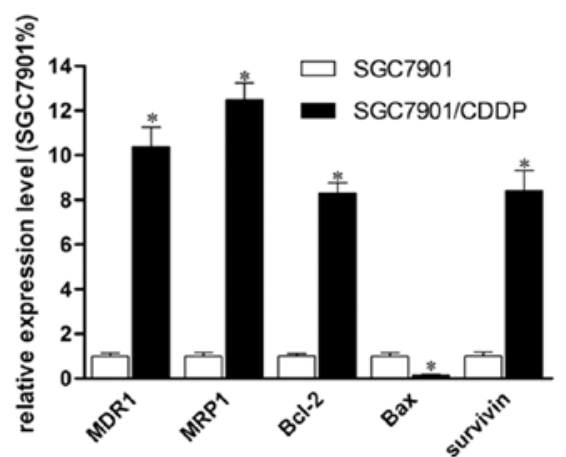

Figure 1. Identification of the gastric carcinoma SGC7901/CDDP cell line to the CDDP-induced MDR phenotype. (A) Cell viability CCK-8 assay. The IC 50 values of SGC7901 and SGC7901/CDDP cells to ADM, 5-FU and CDDP were determined by using CCK-8 assay as described in Materials and methods. ${ }^{*} \mathrm{P}<0.05$ compared to parental SGC7901 cells. (B) Real-time PCR. Relative quantification of MDR1, MRP1, Bcl-2, Bax and survivin mRNA in SGC7901 and SGC7901/CDDP cells was conducted using real-time RT-PCR. For each gene, the data were normalized to the levels of GAPDH expression as relative mRNA level. "P<0.01 compared to parental SGC7901 cells. Data shown are representative of 3 independent experiments. Statistical analysis was performed by the Student's t-test. MDR, multiple drug resistance; ADM, adriamycin; 5-FU, 5-fluorouracil. CDDP, cisplatin.
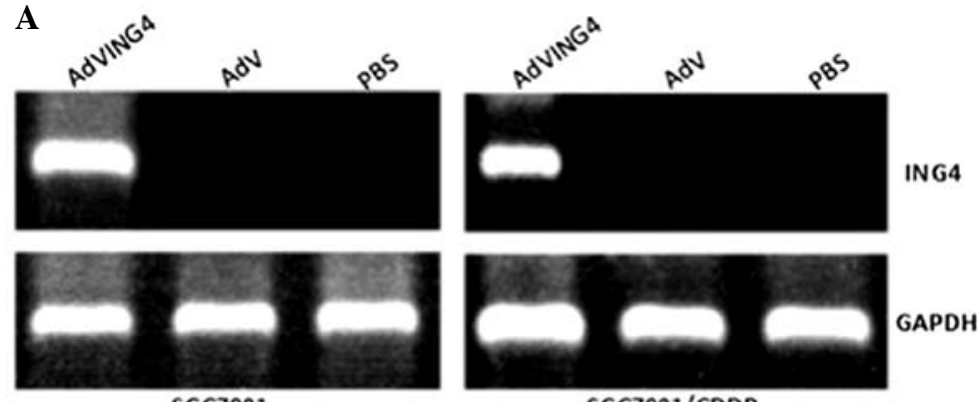

B
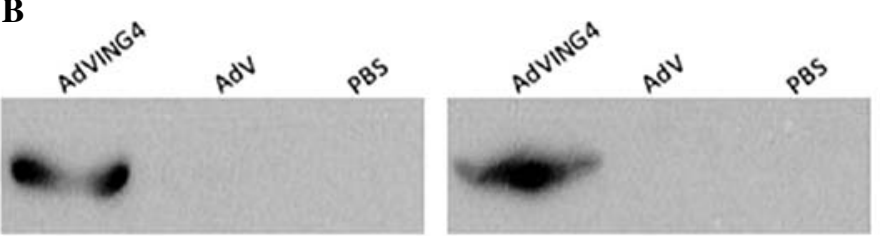

ING4
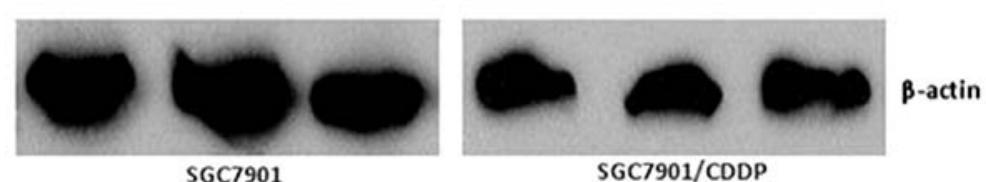

Figure 2. Expression of ING4 mRNA and protein following AdVING4 infection in SGC7901 and SGC7901/CDDP cells. (A) RT-PCR. Total RNA was isolated from AdVING4- or AdV-infected and uninfected SGC7901 and SGC7901/CDDP cells and subjected to RT-PCR. A housekeeping gene GAPDH was used as an internal control. (B) Western blotting. Total cellular lysates of AdVING4- or AdV-infected and uninfected SGC7901 and SGC7901/CDDP cells were analyzed by immunoblotting with an anti-ING4 or anti- $\beta$-actin antibody. Data shown are representative of 3 independent experiments. CDDP, cisplatin.

the SGC7901/CDDP cells when compared to the parental SGC7901 cells (Fig. 1B).

Establishment of stable ING4-expressing gastric cancer SGC7901 cells. To select the optimal MOI for a maximal transgene expression with minimal adenovirus itself-induced cytotoxicity, human gastric carcinoma SGC7901 and SGC7901/CDDP cells were infected with GFP-expressing AdVING4 or AdV at different MOIs and observed using fluorescence microscopy. More than $90 \%$ of GFP expression was found in the AdVING4- or AdV-infected SGC7901 and
SGC7901/CDDP tumor cells at an MOI of 100 or above, whereas the GFP expression was not found in the uninfected control SGC7901 and SGC7901/CDDP tumor cells. Additionally, an adenovirus-elicited cytotoxic effect was rarely noted in the 100 MOI blank AdV-infected SGC7901 and SGC7901/CDDP cells (data not shown). Furthermore, the adenovirus-mediated exogenous ING4 tumor-suppressor gene was significantly expressed in 100 MOI AdVING4infected SGC7901 and SGC7901/CDDP tumor cells but not in the AdV-infected and uninfected SGC7901 and SGC7901/CDDP control cells (Fig. 2A and B), indicating 
A

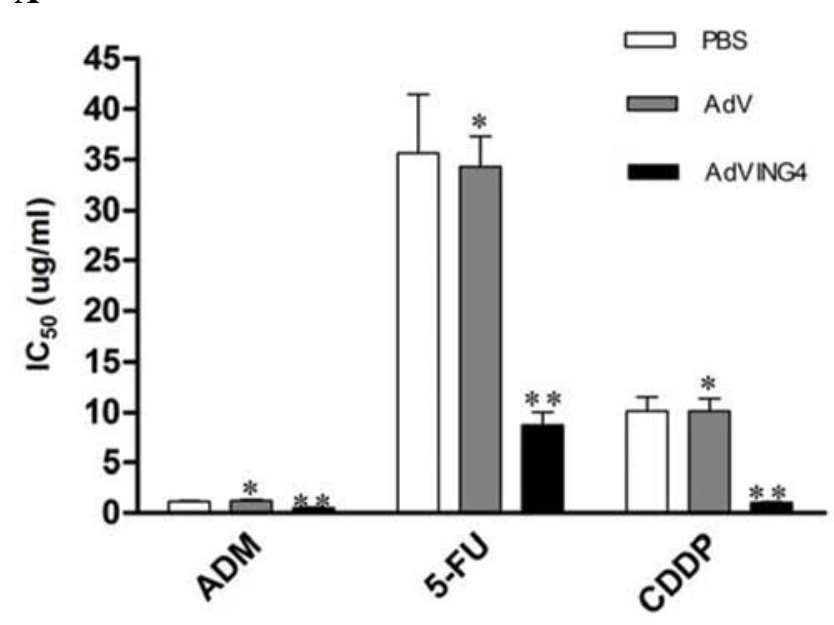

C

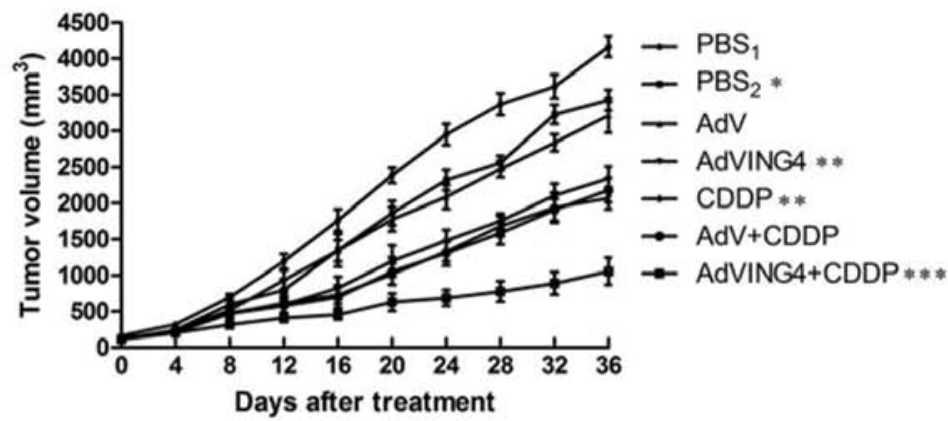

B

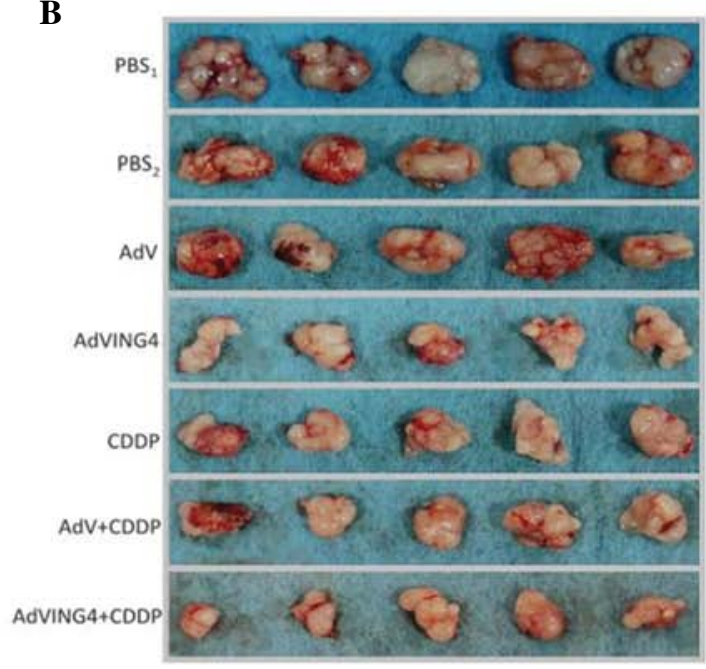

D

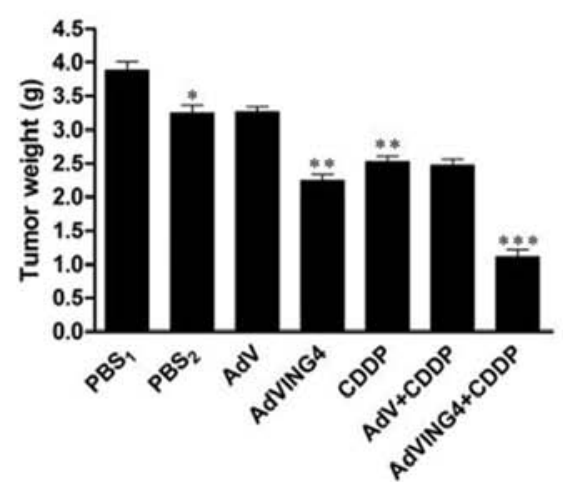

Figure 3. Effects of AdVING4 on the modulation of MDR in SGC7901/CDDP cells in vitro and in vivo. (A) Cell viability CCK-8 assay. The IC s0 $_{5}$ values of SGC7901/CDDP cells infected with AdV or AdVING4 and then treated with ADM, 5-FU and CDDP were evaluated by CCK-8. "P $>0.05$ compared to the PBS control cells, ${ }^{* *} \mathrm{P}<0.05$ compared to PBS and AdV groups, respectively, using one-way repeated ANOVA measures and multiple comparisons. $\mathrm{n}=3$ replicates/condition. (B) Nude mouse xenograft assay. Images of tumor masses in the different groups. (C and D) Tumor volume and weight. The athymic nude mice bearing gastric carcinoma SGC7901/CDDP cell xenograft tumors were intratumoral injected with AdVING4 (1x10 ${ }^{8}$ GTU), AdV (1x10 GTU), PBS or CDDP

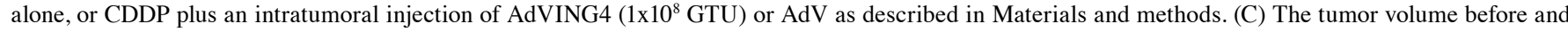
after treatment and (D) the tumor weight at 36 days after treatment. $\mathrm{P}<0.05$ compared to $\mathrm{PBS}_{1}$ group; ${ }^{* *} \mathrm{P}<0.05$ compared to $\mathrm{PBS}_{2}$ and $\mathrm{AdV}$ groups; ${ }^{* * * *} \mathrm{P}<0.05$ compared to AdVING4, CDDP and AdV+CDDP groups, respectively $\left(1 \times 10^{8} \mathrm{GTU}\right)$ using one-way and two-way repeated ANOVA measures and multiple comparisons ( $\mathrm{n}=5$ mice/condition). Data shown are representative of 3 independent experiments. The data showed that AdVING4 reversed MDR and consequently AdVING4 plus CDDP induced synergistic tumor inhibition in vivo. MDR, multiple drug resistance; ADM, adriamycin; 5-FU, 5-fluorouracil; CDDP, cisplatin.

that the ING4 tumor-suppressor gene was lost or profoundly downregulated in the human gastric carcinoma SGC7901 and SGC7901/CDDP cells. The results suggest that $100 \mathrm{MOI}$ can be used as an optimal dose for the adenovirus-directed ING4 gene induction and transgene expression in human gastric carcinoma SGC7901 and SGC7901/CDDP cells.

AdVING4 infection reverses the MDR of SGC7901/CDDP cells in vitro and in vivo. Based on the CDDP-induced MDR phenotype in SGC7901/CDDP cells, we first investigated the AdVING4-mediated reversal effects of AdVING4 infection on SGC7901/CDDP tumor cells in vitro. Gastric carcinoma SGC7901/CDDP cells were infected with AdVING4 or AdV at an MOI of 100, and tumor cell viability was determined on day 4 using the CCK-8 assay. The $\mathrm{IC}_{50}$ of the AdVING4infected SGC7901/CDDP cells was significantly decreased, whereas this phenomenon did not occur in the $\mathrm{AdV}$ - or PBS-treated cells $(\mathrm{P}<0.05)$ (Fig. 3A), indicating that AdVING4 is a potent modulator for the CDDP-induced MDR phenotype in gastric carcinoma SGC7901/CDDP cells. To further address the potential effects of AdVING4 on SGC7901/CDDP cells in vivo, we injected SGC7901/CDDP cells into athymic nude mice and further injected AdVING4, AdV (1x10 8 GTU), or PBS followed by treatment with or without CDDP. The data showed that AdVING4 plus CDDP significantly reduced the tumor volume from days 12 to 36 when compared to the other groups $(\mathrm{P}<0.05$; Fig. 3B and $\mathrm{C})$. Similarly, the tumor weight was also reduced in the AdVING4 plus CDDP-treated mice (Fig. 3D), indicating that AdVING4 markedly modulated MDR of gastric carcinoma SGC7901/CDDP cell xenografts in vivo in the athymic nude mouse model.

Effect of AdVING4 on MDR and expression of apoptosisrelated genes. To further address the underlying molecular events that may be responsible for the AdVING4-mediated effects in reversing MDR, expression levels of MDR-related genes (MDR1 and MRP1) and apoptosis-related genes (Bcl-2, Bax and survivin) in these cells were analyzed. The data showed that levels of MDR1, MRP1, Bcl-2 and survivin were apparently downregulated, whereas expression of apoptosis-promoting 


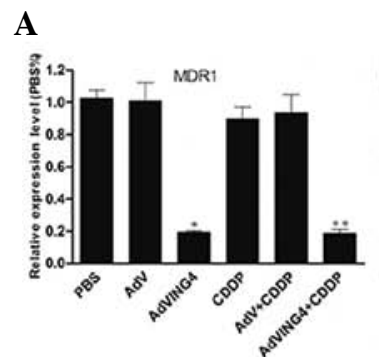

$\mathbf{B}$
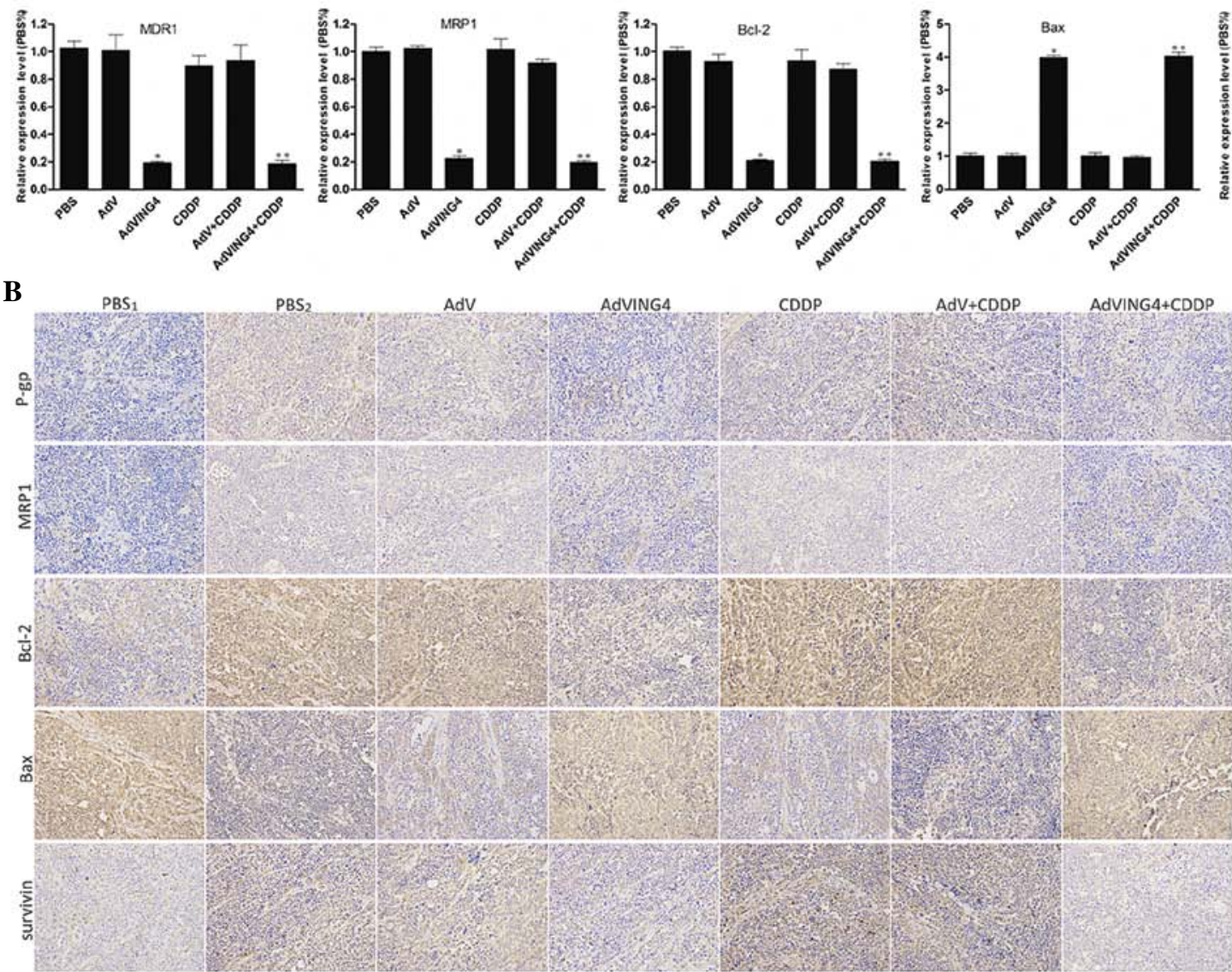
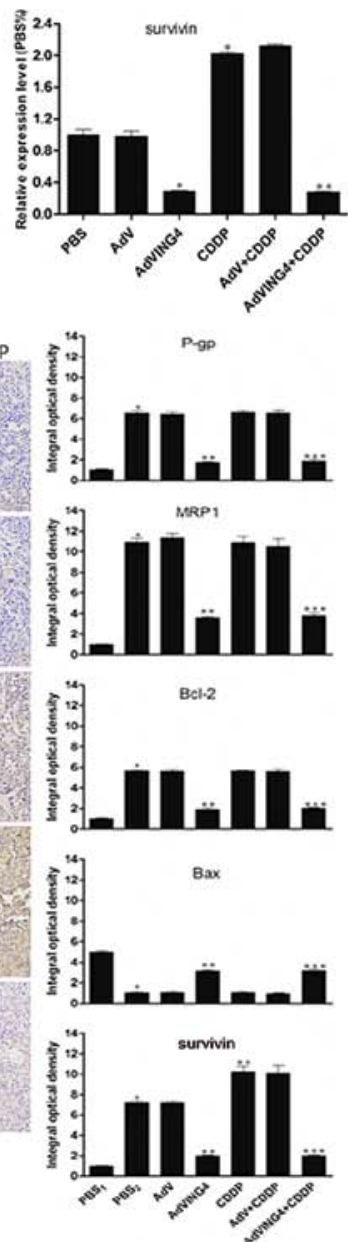

Figure 4. Effect of AdVING4 on the expression of MDR-related and apoptosis-related genes. (A) qRT-PCR. Relative quantification of MDR1, MRP1, Bcl-2, Bax and survivin mRNA in cells following various treatments was analyzed using qRT-PCR. The data were normalized to levels of GAPDH expression. ${ }^{*} \mathrm{P}<0.01$ compared to PBS and AdV cells; ${ }^{* *} \mathrm{P}<0.01$ compared to CDDP and AdV+CDDP-treated cells; ${ }^{* * *} \mathrm{P}>0.05$ compared to AdVING4-infected cells by using one-way repeated ANVOA measures and multiple comparisons ( $\mathrm{n}=3$ replicates/condition, $\mathrm{n}=2 \mathrm{replicates} / \mathrm{sample})$. (B) Representative images of immunohistochemical detection of P-gp, MRP1, Bcl-2, Bax and survivin in gastric carcinoma SGC7901/CDDP xenograft tissues. Consequently, the integral optical density (IOD) of the immunostaining intensity was quantified using Image-Pro Plus 6.0 software. ${ }^{*} \mathrm{P}<0.05$ compared to $\mathrm{PBS}_{1}$; ${ }^{* *} \mathrm{P}<0.05$ compared to $\mathrm{PBS} \mathrm{S}_{2}$ and AdV groups; ${ }^{* * *} \mathrm{P}<0.05$ compared to CDDP and AdV+CDDP groups; ${ }^{* * *} \mathrm{P}>0.05$ compared to AdVING4 group by using one-way repeated ANOVA measures and multiple comparisons ( $n=6$ replicates/condition, $n=5$ observations/representative section). Original magnification, $x 400$. Data shown are representative of 3 independent experiments. MDR, multiple drug resistance; CDDP, cisplatin.

gene, Bax, was upregulated in AdVING4 and AdVING4 plus CDDP-treated SGC7901/CDDP cells compared to the PBS, $\mathrm{AdV}, \mathrm{CDDP}$ or AdV plus CDDP groups $(\mathrm{P}<0.01)$. Furthermore, expression of MDR-related proteins, P-gp and MRP1, and apoptosis-related proteins $\mathrm{Bcl}-2$, Bax and survivin was also modulated in the nude mouse xenograft tissues $(\mathrm{P}<0.05$; Fig. 4A and B). The results indicate that AdVING4 may play an important role in reversing MDR in SGC7901/CDDP cells by regulating expression of $\mathrm{P}$-gp, MRP1, Bcl-2, Bax and survivin.

\section{Discussion}

In the present study, we investigated the MDR of CDDP-induced gastric cancer cells and found that MDR promoted the resistance of gastric cancer cells 40-fold to CDDP treatment or other chemotherapeutic drugs, including 5-FU or ADM. We further determined the effects of AdVING4-induced ING4 expression on these tumor cells and found that induction of
ING4 expression significantly sensitized these gastric cancer cells to chemotherapy in vitro and in nude mouse xenografts. At the molecular level, ING4 expression inhibited expression of MDR-related proteins (P-gp and MRP1) and apoptosissuppressing proteins (Bcl-2 and survivin), but induced expression of pro-apoptosis Bax protein in vitro and in tumor xenograft tissues. These data indicate that AdVING4 warrants further verification as a feasible adjuvant chemotherapy for gastric carcinoma patients.

Clinically, the effectiveness of chemotherapy on gastric cancer or other types of cancer is modest or disappointing as evidenced by tumor cell chemoresistance, while a highdose treatment of chemotherapeutic drugs induces severe side-effects, such as an immunocompromised state, bone marrow suppression, digestive disturbances, renal toxicity, ototoxicity or ascites. Although much effort has been made to explore the causes of tumor chemoresistance, the underlying molecular mechanisms responsible for chemoresistance 
remain uncler. Previously studies have shown that MDR is a major cause for the failure of many modes of chemotherapy (8) and limits the efficacy of cancer treatment. Upregulated expression of ATP-binding cassette transporters, such as P-gp and MRP1, may play a central role in acquired drug resistance as these proteins act as 'pumps' to lower the intracellular drug concentration and reduce drug efficacy in tumor cells (30). Therefore, since the early 1980's, various compounds have been identified to overcome P-gp- and MRP1-mediated MDR. However, they have achieved only limited success in clinical trials. Therefore, the characterization of signaling pathways sustaining MDR is thus essential for designing rational novel therapies for MDR. Recently, cancer gene therapy represents a promising novel therapeutic modality for cancers, particularly for the treatment of cancers of the digestive system (31). Various strategies combining gene or molecular therapy with chemotherapy have been applied to radiosensitize or chemosensitize tumor cells to chemotherapy or radiation therapy and to reduce treatment-associated toxicity $(25,26,32)$. To date limited success has been reported. For example, the adenoviral p53 gene has been in adjuvant use together with conventional chemotherapy, radiation therapy, and surgery for lung, and head and neck cancers (33).

In the present study, we focused on the ING4 gene as ING4 is a tumor-suppressor gene and plays a role in suppression of oncogenesis, tumor cell growth, angiogenesis, migration and gene transcription. A number of studies have shown that ING4 expression is frequently downregulated or deleted in a large variety of cancer, including gastric cancer (22,34-37). Moreover, restoration of ING4 expression was found to suppress tumor growth in a p53-dependent and p53-independent manner, induce tumor cell cycle arrest and apoptosis $(13,19-21)$, and inhibit tumor angiogenesis by repressing nuclear factor- $\kappa \mathrm{B}$ and hypoxia-inducible factor-1 $\alpha$ (22-24). ING4 protein can also enhance radiosensitivity and chemosensitivity in nonsmall-cell lung cancer and hepatocarcinoma cells $(19,25)$. In the present study, we first successfully established the CDDPinduced MDR phenotype in a gastric carcinoma cell line for assessing the effects of the AdVING4 gene on the reversion of the MDR phenotype in gastric carcinoma cells in vitro and in vivo. We demonstrated that AdVING4-mediated ING4 expression decreased the $\mathrm{IC}_{50}$ of CDDP, 5-FU and ADM, accelerated apoptosis in the CDDP-induced MDR phenotype of gastric carcinoma cells. Moreover, AdVING4 plus CDDP also synergistically inhibited the growth of gastric carcinoma xenograft tumors in athymic nude mice. This finding suggests that ING4 may be useful for the clinical adjuvant therapy of gastric cancer.

Furthermore, the results showed that AdVING4 was able to inhibit expression of P-gp, MRP1, Bcl-2 and survivin while increasing Bax expression in vitro and in vivo, which molecularly supports that ING4 could regulate the CDDP-induced MDR of gastric cancer cells. Previous studies have shown that $\mathrm{P}$-gp is only expressed in the proximal tubular epithelial cells of normal human kidney and not in other cell types (38), but P-gp expression is enhanced by long-term chemotherapy in tumor cells (39). Thus, suppression of P-gp expression could sensitize tumor cells to chemotherapy (40). Indeed, the present study showed that P-gp was significantly increased in CDDPinduced MDR gastric carcinoma cells and that overexpression of ING4 protein significantly decreased P-gp expression and AdVING4 plus CDDP induced significant apoptosis in gastric carcinoma xenografts in athymic nude mice. This finding demonstrated that $\mathrm{P}$-gp protein could mediate MDR in gastric cancer cells. Moreover, MRP1 protein is usually localized in proximal tubular epithelial cells of normal renal tissue, and the basolateral localization of the protein is important for efflux of its substrates into blood (41). Another study showed that MRP1 expression was significantly correlated with the survival of patients with neuroblastoma (42). The present study showed that MRP1 expression was also upregulated following CDDP-induced MDR in SGC7901/CDDP cells, while AdVING4 downregulated MRP1 expression in the gastric carcinoma cells in vitro and in vivo. Molecularly, ING4 is involved in the p53-dependent regulatory pathway for its antitumor activity (19), and a previous study showed that p53 plays a decisive role in the regulation of P-gp and MRP1 expression in chemoresistance $(43,44)$. Thus, the downregulation of P-gp and MRP1 expression by ING4 may be through a p53-dependent pathway.

In addition, the $\mathrm{Bcl}-2$ protein family is known to be a pivotal regulator of apoptosis and an important determinant of cellular fate (45). A previous study showed that apoptosis-related genes (such as Bax, Bcl-2 and survivin) are key factors responsible for MDR $(25,32,46)$. The ratio of anti- to pro-apoptotic Bcl-2 family molecules, such as Bcl-2/Bax, constitutes a rheostat that sets the threshold of susceptibility to apoptosis for the intrinsic apoptosis pathway, which promotes pore formation in the mitochondrial outer membrane, loss of mitochondrial integrity, and the release into the cytosol of cytochrome $c$ followed by the cleavage of caspase-9, leading to the activation of the intrinsic apoptotic pathway. Meanwhile, survivin can inhibit apoptosis by interacting with cyclin kinase cdk4, p34 and cdc2 and block apoptotic signal transduction. Survivin is barely expressed in normal adult tissues but is weakly expressed in the placenta and thymus (47); however, it is widely expressed in tumor cells (48). Expression of survivin in cancer was shown to be closely associated with malignant phenotypes, poor prognosis, tumor recurrence and drug resistance (47). In the present study, Bcl-2 and survivin mRNA and protein were significantly upregulated while Bax was downregulated in the SGC7901/CDDP cells and xenograft tumors when compared to their levels in the parental cells. AdVING4 elicited an encouraging effect on the upregulation of Bax but downregulated Bcl-2 and survivin expression to activate the apoptotic pathways. However, the present study is just a proof-of-principle, and further study is required to investigate the safety issue of AdVING4 for clinical use in cancer patients. In addition, we will investigate the underlying molecular gene pathways that mediated ING4-reduced MDR in gastric cancer cells.

\section{Acknowledgements}

This study was supported by the Project of the Nature Science Foundation of China (81201905), the Shanghai Postdoctoral Scientific Program of China (13R21415200), the Nature Science Research Grants in University of Jiangsu Province of China (12KJB320009) and the Science and Technology Research Project of in Science and Technology Bureau of Suzhou City of China (YJS0916 and SYS201220). 


\section{References}

1. Forman D and Burley VJ: Gastric cancer: global pattern of the disease and an overview of environmental risk factors. Best Pract Res Clin Gastroenterol 20: 633-649, 2006.

2. Roder DM: The epidemiology of gastric cancer. Gastric Cancer 5 (Suppl 1): S5-S11, 2002.

3. Shin HR, Jung KW, Won YJ, Park JG; 139 KCCR-affiliated Hospitals: 2002 annual report of the Korea Central Cancer Registry: based on registered data from 139 hospitals. Cancer Res Treat 36: 103-114, 2004.

4. Kelley JR and Duggan JM: Gastric cancer epidemiology and risk factors. J Clin Epidemiol 56: 1-9, 2003.

5. Sun XD, Mu R, Zhou YS, et al: Analysis of mortality rate of stomach cancer and its trend in twenty years in China. Zhonghua Zhong Liu Za Zhi 26: 4-9, 2004 (In Chinese).

6. Cunningham D, Allum WH, Stenning SP, et al: Perioperative chemotherapy versus surgery alone for resectable gastroesophageal cancer. N Engl J Med 355: 11-20, 2006.

7. Rivera F, Vega-Villegas ME and López-Brea MF: Chemotherapy of advanced gastric cancer. Cancer Treat Rev 33: 315-324, 2007.

8. Arceci RJ: Tumor cell survival and resistance to therapy. Curr Opin Hematol 3: 279-287, 1996.

9. Filipits M: Mechanisms of cancer: multidrug resistance. Drug Discov Today Dis Mech 1: 229-234, 2004.

10. Xia L, Zhang D, Du R, et al: miR-15b and miR-16 modulate multidrug resistance by targeting BCL2 in human gastric cancer cells. Int J Cancer 123: 372-379, 2008.

11. Shi Y, Zhai H, Wang X, et al: Ribosomal proteins $\mathrm{S} 13$ and L23 promote multidrug resistance in gastric cancer cells by suppressing drug-induced apoptosis. Exp Cell Res 296: 337-346, 2004.

12. Zhao Y, You H, Liu F, et al: Differentially expressed gene profiles between multidrug resistant gastric adenocarcinoma cells and their parental cells. Cancer Lett 185: 211-218, 2002.

13. Shiseki M, Nagashima M, Pedeux RM, et al: p29ING4 and p28ING5 bind to $\mathrm{p} 53$ and $\mathrm{p} 300$, and enhance p53 activity. Cancer Res 63: 2373-2378, 2003.

14. He GH, Helbing CC, Wagner MJ, Sensen CW and Riabowol K: Phylogenetic analysis of the ING family of PHD finger proteins. Mol Biol Evol 22: 104-116, 2005.

15. Aasland R, Gibson TJ and Stewart AF: The PHD finger: implications for chromatin-mediated transcriptional regulation. Trends Biochem Sci 20: 56-59, 1995.

16. Zhang X, Wang KS, Wang ZQ, et al: Nuclear localization signal of ING4 plays a key role in its binding to p53. Biochem Biophys Res Commun 331: 1032-1038, 2005.

17. Kim S, Chin K, Gray JW and Bishop JM: A screen for genes that suppress loss of contact inhibition: identification of ING4 as a candidate tumor suppressor gene in human cancer. Proc Natl Acad Sci USA 101: 16251-16256, 2004.

18. Kim S, Welm AL and Bishop JM: A dominant mutant allele of the ING4 tumor suppressor found in human cancer cells exacerbates $M Y C$-initiated mouse mammary tumorigenesis. Cancer Res 70: 5155-5162, 2010.

19. Zhang X, Xu LS, Wang ZQ, et al: ING4 induces G2/M cell cycle arrest and enhances the chemosensitivity to DNA-damage agents in HepG2 cells. FEBS Lett 570: 7-12, 2004.

20. Unoki M, Shen JC, Zheng ZM and Harris CC: Novel splice variants of ING4 and their possible roles in the regulation of cell growth and motility. J Biol Chem 281: 34677-34686, 2006.

21. Xie Y, Zhang H, Sheng W, Xiang J, Ye Z and Yang J: Adenovirusmediated ING4 expression suppresses lung carcinoma cell growth via induction of cell cycle alteration and apoptosis and inhibition of tumor invasion and angiogenesis. Cancer Lett 271: 105-116, 2008.

22. Garkavtsev I, Kozin SV, Chernova O, et al: The candidate tumour suppressor protein ING4 regulates brain tumour growth and angiogenesis. Nature 428: 328-332, 2004.

23. $\mathrm{Li} \mathrm{J}$ and Li G: Cell cycle regulator ING4 is a suppressor of melanoma angiogenesis that is regulated by the metastasis suppressor BRMS1. Cancer Res 70: 10445-10453, 2010.

24. Colla S, Tagliaferri S, Morandi F, et al: The new tumor-suppressor gene inhibitor of growth family member 4 (ING4) regulates the production of proangiogenic molecules by myeloma cells and suppresses hypoxia-inducible factor-1 $\alpha$ (HIF-1 $\alpha)$ activity: involvement in myeloma-induced angiogenesis. Blood 110: 4464-4475, 2007.
25. Ling C, Xie Y, Zhao D, Zhu Y, Xiang J and Yang J: Enhanced radiosensitivity of non-small-cell lung cancer (NSCLC) by adenovirus-mediated ING4 gene therapy. Cancer Gene Ther 19: 697-706, 2012.

26. Xie Y, Sheng W, Miao J, Xiang J and Yang J: Enhanced antitumor activity by combining an adenovirus harboring ING4 with cisplatin for hepatocarcinoma cells. Cancer Gene Ther 18: 176-188, 2011.

27. Nishikawa T, Ramesh R, Munshi A, Chada S and Meyn RE: Adenovirus-mediated mda-7 (IL24) gene therapy suppresses angiogenesis and sensitizes NSCLC xenograft tumors to radiation. Mol Ther 9: 818-828, 2004.

28. Zhang X, Cheung RM, Komaki R, Fang B and Chang JY: Radiotherapy sensitization by tumor-specific TRAIL gene targeting improves survival of mice bearing human non-small cell lung cancer. Clin Cancer Res 11: 6657-6668, 2005.

29. Livak KJ and Schmittgen TD: Analysis of relative gene expression data using real-time quantitative PCR and the $2^{-\triangle \Delta C T}$ method. Methods 25: 402-408, 2001.

30. Kong D, Ma S, Liang B, et al: The different regulatory effects of p53 status on multidrug resistance are determined by autophagy in ovarian cancer cells. Biomed Pharmacother 66: 271-278, 2012.

31. Touchefeu Y, Harrington KJ, Galmiche JP and Vassaux G: Review article: gene therapy, recent developments and future prospects in gastrointestinal oncology. Aliment Pharmacol Ther 32: 953-968, 2010.

32. Zhu W, Shan X, Wang T, Shu Y and Liu P: miR-181b modulates multidrug resistance by targeting BCL2 in human cancer cell lines. Int J Cancer 127: 2520-2529, 2010.

33. Moon C, Oh Y and Roth JA: Current status of gene therapy for lung cancer and head and neck cancer. Clin Cancer Res 9: 5055-5067, 2003 .

34. Gunduz M, Nagatsuka H, Demircan K, et al: Frequent deletion and down-regulation of ING4, a candidate tumor suppressor gene at 12 p13, in head and neck squamous cell carcinomas. Gene 356: 109-117, 2005.

35. Fang F, Luo LB, Tao YM, Wu F and Yang LY: Decreased expression of inhibitor of growth 4 correlated with poor prognosis of hepatocellular carcinoma. Cancer Epidemiol Biomarkers Prev 18: 409-416, 2009.

36. Wang QS, Li M, Zhang LY, et al: Down-regulation of ING4 is associated with initiation and progression of lung cancer. Histopathology 57: 271-281, 2010.

37. Tapia C, Zlobec I, Schneider S, et al: Deletion of the inhibitor of growth 4 (ING4) tumor suppressor gene is prevalent in human epidermal growth factor 2 (HER2)-positive breast cancer. Hum Pathol 42: 983-990, 2011.

38. Hodorová I, Rybárová S, Vecanová J, Solár P, Plank L and Mihalik J: Relation between expression pattern of wild-type p53 and multidrug resistance proteins in human nephroblastomas. Acta Histochem 115: 273-278, 2013.

39. Camassei FD, Arancia G, Cianfriglia M, et al: Nephroblastoma: multidrug-resistance P-glycoprotein expression in tumor cells and intratumoral capillary endothelial cells. Am J Clin Pathol 117: 484-490, 2002.

40. Yin F, Shi YQ, Zhao WP, Xiao B, Miao JY and Fan DM: Suppression of P-gp induced multiple drug resistance in a drug resistant gastric cancer cell line by overexpression of Fas. World $\mathbf{J}$ Gastroenterol 6: 664-670, 2000.

41. Sarkadi B, Ozvegy-Laczka C, Német K and Váradi A: ABCG2 a transporter for all seasons. FEBS Lett 567: 116-120, 2004.

42. Norris MD, Bordow SB, Marshall GM, Haber PS, Cohn SL and Haber M: Expression of the gene for multidrug-resistanceassociated protein and outcome in patients with neuroblastoma. N Engl J Med 334: 231-238, 1996.

43. Sakaeda T, Nakamura T, Horinouchi M, et al: MDR1 genotype-related pharmacokinetics of digoxin after single oral administration in healthy Japanese subjects. Pharm Res 18: 1400-1404, 2001.

44. Hait WN and Yang JM: The individualization of cancer therapy: the unexpected role of p53. Trans Am Clin Climatol Assoc 117: 85-101, 2006.

45. Danial NN and Korsmeyer SJ: Cell death: critical control points. Cell 116: 205-219, 2004

46. Li K, Lu Y, Liang J, et al: RhoE enhances multidrug resistance of gastric cancer cells by suppressing Bax. Biochem Biophys Res Commun 379: 212-216, 2009.

47. Adida C, Crotty PL, McGrath J, Berrebi D, Diebold J and Altieri DC: Developmentally regulated expression of the novel cancer anti-apoptosis gene survivin in human and mouse differentiation. Am J Pathol 152: 43-49, 1998.

48. Altieri DC: The molecular basis and potential role of survivin in cancer diagnosis and therapy. Trends Mol Med 7: 542-547, 2001. 